\title{
Asymptotic behavior of solutions of nonlinear models from erythropoiesis
}

\section{Xiao Liang*}

Correspondence: xili09@126.com School of Basic Medical Sciences, Fudan University, Shanghai, 200032, China

\begin{abstract}
We consider a class of nonlinear models associated with erythropoiesis and establish a global asymptotic stability result for the trivial steady state, which extends essentially some previous results. Moreover, we give numerical simulations to illustrate this theoretical result.
\end{abstract}

Keywords: nonlinear models; asymptotic behavior of solutions; erythropoiesis

\section{Introduction}

Erythropoiesis is the process by which red blood cells (erythrocytes) are formed. It is a complex process, stimulated by decreased $\mathrm{O}_{2}$ in circulation. Detecting this decrease, the kidneys then secrete the hormone erythropoietin. This hormone stimulates proliferation and differentiation of red cell precursors, which activates increased erythropoiesis in the hemopoietic tissues, ultimately producing red blood cells. This process (erythropoiesis) is based upon the differentiation of the hematopoietic stem cells. The hematopoietic stem cells are undifferentiated cells in a self-maintained stem cell compartment (located in the bone morrow), which are either proliferating or nonproliferating cells and have unique capacities of producing cells committed to one of the three blood cell types: red blood cells, white cells or platelets, and self-renewal. Erythrocytes (red blood cells) carry out the exchange of oxygen and carbon dioxide between the lungs and the body tissues. To effectively combine with oxygen, the erythrocytes must contain a normal amount of the red protein pigment hemoglobin ( $c f$. [1]), the amount of which in turn depends on the iron level in the body. Erythrocytes are biconcave in shape, which increases the cell's surface area and facilitates the diffusion of oxygen and carbon dioxide. From [2] and the related literature, one can get to know the following information on erythrocytes. Erythrocytes are produced primarily from the $\mathrm{CD} 34^{+}$pluripotent hematopoietic stem cells of bone marrow. $\mathrm{CD} 34^{+}$progenitors are isolated from adult peripheral blood or cord blood and grown in liquid medium in fibronectin-coated wells. These progenitor stem cells constitute approximately $0.1 \%$ of nucleated cells in the bone marrow, only about $5 \%$ of which are in cycle at any one time. Cell differentiation along the erythroid lineage occurs over a two-week span in humans. The earliest erythroid progenitor, the BFU-E (burst forming unit-erythroid), is small and without distinguishing histologic characteristics. BFU-Es express the cell surface antigen, CD34, as do all other early hematopoietic progenitors. The stage after the BFU-E is the CFU-E (colony forming unit-erythroid), which is larger and is the stage right before hemoglobin production begins. Immature erythroblasts, which 
start producing hemoglobin, also start condensing their nuclei. Mature erythroblasts are smaller with tightly compacted nuclei which are expelled as the cells become reticulocytes. Cell division ceases with the formation of the orthochromatic erythroblast. Division rate, death rate, and maturation rate are influenced by the level of erythropoietin. The erythrocyte lineage shares the precursor CFU-GEMM (granulocyte, erythrocyte, macrophage, megakaryocyte) with other types of blood cells (white blood cells, platelets, etc.). The process of erythropoiesis has been modeled in many physiological scenarios. For more information about erythropoiesis and the differentiation of the hematopoietic stem cells, we refer the reader to [1-6] and references therein.

To the best of our knowledge, the first mathematical model of hematopoietic stem cells' dynamics was established by Mackey [4], which is an uncoupled system of two nonlinear delay differential equations, describing the dynamics of proliferating and nonproliferating hematopoietic stem cells. The time delay, which is constant, accounts for an average cell cycle duration. Since then, Mackey's model has been improved and studied by many authors ( $c f$. [3, 5-10]). Stimulated and inspired by these works, especially by [7], we investigate the following general nonlinear system with time delay $\tau$, corresponding to the cell cycle duration where the cells in cycle are divided only in two groups: proliferating and nonproliferating cells,

$$
\begin{aligned}
& S^{\prime}(t)=-\delta S(t)+e^{-\delta \tau} \beta(S(t-\tau)) f(N(t-\tau)), \quad t \geq 0, \\
& N^{\prime}(t)=-\delta N(t)-\beta(S(t)) f(N(t))+2 e^{-\delta \tau} \beta(S(t-\tau)) f(N(t-\tau)), \quad t \geq 0 .
\end{aligned}
$$

Here, $S(t)$ and $N(t)$ stand for the total number of hematopoietic stem cells and nonproliferating cells at time $t$ respectively, $\delta>0$ is the rate of differentiation of nonproliferating cells, $\tau$ is the average duration of the proliferating phase and the term $e^{-\delta \tau}$ then describes the survival rate of proliferating cells, $\beta(\cdot)$ is a continuously differentiable, positive, and decreasing function with

$$
\lim _{x \rightarrow+\infty} \beta(x)=0
$$

denoting the rate of cell movement from nonproliferating cell compartment into the proliferation, which depends on the total number of hematopoietic stem cells, and $f(\cdot)$ is a continuously differentiable function with

$$
0 \leq f(x) \leq L x \quad(\forall x \geq 0), \quad f(x)>0 \quad(\forall x>0)
$$

( $L$ is a positive constant) describing the nonlinear change of the number of nonproliferating cell compartments. This nonlinear system is more general than those in the previous literature since we take the change (being nonlinear in many cases) of the number of nonproliferating cell compartments into account. A typical example of $f$ is the following function:

$$
f(x)=\frac{\mu x}{k+x^{r}}
$$

where $\mu>0, k>0$, and $r>1$ are constants. This is a single-humped function of $x$, which was first considered for modeling the hematopoietic stem cells' dynamics in [6] by Mackey 
and Glass. With such a nonlinear term, the study of system (1.1)-(1.2), which is a generalized Mackey-Glass-type model, is more complicated than that of previous models. The purpose of the paper is to establish a global asymptotic stability result for the trivial steady state of this class of systems, which shows that in many cases, the hematopoietic stem cell population is definitely extinct. The next section is devoted to proving this criterion. In the last section, we give numerical simulations to illustrate this phenomenon.

\section{Asymptotic behavior of solutions}

It follows from many books on delay equations (e.g., [9]) that for each continuous initial condition, system (1.1)-(1.2) has a unique continuous solution $(S(t), N(t))$ for $t \geq 0$. For more information on delay equations, see, e.g., [7, 11-15].

Theorem 2.1 Let $(S(t), N(t))$ be a solution of (1.1)-(1.2) for a positive initial datum. Then $S(t)$ and $N(t)$ are positive for $t \geq 0$.

Proof To obtain the required conclusion, we will show that if $(S(t), N(t))$ is a solution of (1.1)-(1.2) for a positive initial datum, then the following situations:

(i) neither $S(t)$ nor $N(t)$ is positive;

(ii) $N(t)$ is positive but not $S(t)$;

(iii) $S(t)$ is positive but not $N(t)$;

do not appear definitely.

Step 1: We show that it is impossible that neither $S(t)$ nor $N(t)$ is positive.

Suppose that neither $S(t)$ nor $N(t)$ is positive. Then $S(t)$ has one zero point in $[0,+\infty)$ at least, and so does $N(t)$. Let $t_{0}$ and $t_{1}$ be the first zero point of $S(t)$ and $N(t)$ in $[0,+\infty)$, respectively. Then, by $S(0)>0$ and $N(0)>0$, we know that $t_{0}>0$ and $t_{1}>0$.

Case 1: $t_{0} \leq t_{1}$.

In this case, by the definition of $t_{0}$ and $t_{1}$, we have

$$
S(t)>0 \quad\left(\forall t \in\left[0, t_{0}\right)\right), \quad S\left(t_{0}\right)=0,
$$

and

$$
N(t)>0 \quad\left(\forall t \in\left[0, t_{0}\right)\right) .
$$

Hence,

$$
S^{\prime}\left(t_{0}\right)=\lim _{h \rightarrow 0^{-}} \frac{S\left(t_{0}+h\right)-S\left(t_{0}\right)}{h}=\lim _{h \rightarrow 0^{-}} \frac{S\left(t_{0}+h\right)}{h} \leq 0 .
$$

Moreover, it follows from (1.2)-(1.4) and the positivity of the initial datum (i.e., $S(t)>0$ and $N(t)>0$ for $t \in[-\tau, 0])$ that

$$
\begin{aligned}
S^{\prime}\left(t_{0}\right) & =-\delta S\left(t_{0}\right)+e^{-\delta \tau} \beta\left(S\left(t_{0}-\tau\right)\right) f\left(N\left(t_{0}-\tau\right)\right) \\
& =e^{-\delta \tau} \beta\left(S\left(t_{0}-\tau\right)\right) f\left(N\left(t_{0}-\tau\right)\right) \\
& >0 .
\end{aligned}
$$

This contradicts (2.1). 
Case 2: $t_{1} \leq t_{0}$.

In this case, by the definition of $t_{0}$ and $t_{1}$, we have

$$
N(t)>0 \quad\left(\forall t \in\left[0, t_{1}\right)\right), \quad N\left(t_{1}\right)=0,
$$

and

$$
S(t)>0 \quad\left(\forall t \in\left[0, t_{1}\right)\right) .
$$

Therefore,

$$
N^{\prime}\left(t_{1}\right)=\lim _{h \rightarrow 0^{-}} \frac{N\left(t_{1}+h\right)-N\left(t_{1}\right)}{h}=\lim _{h \rightarrow 0^{-}} \frac{N\left(t_{1}+h\right)}{h} \leq 0 .
$$

Furthermore, (1.2)-(1.4) and the positivity of the initial datum (i.e., $S(t)>0$ and $N(t)>0$ for $t \in[-\tau, 0])$ imply that

$$
\begin{aligned}
N^{\prime}\left(t_{1}\right) & =-\delta N\left(t_{1}\right)-\beta\left(S\left(t_{1}\right)\right) f\left(N\left(t_{1}\right)\right)+2 e^{-\delta \tau} \beta\left(S\left(t_{1}-\tau\right)\right) f\left(N\left(t_{1}-\tau\right)\right) \\
& =2 e^{-\delta \tau} \beta\left(S\left(t_{1}-\tau\right)\right) f\left(N\left(t_{1}-\tau\right)\right) \\
& >0 .
\end{aligned}
$$

This contradicts (2.3).

Consequently, it is impossible that neither $S(t)$ nor $N(t)$ is positive.

Step 2: We prove that it is impossible that $N(t)$ is positive but not $S(t)$.

Suppose that $N(t)$ is positive but not $S(t)$. Then $S(t)$ has one zero point in $[0,+\infty)$ at least. Let $t_{0}$ be the first zero point of $S(t)$ in $[0,+\infty)$. Then $S(0)>0$ implies that $t_{0}>0$ and

$$
S(t)>0 \quad\left(\forall t \in\left[0, t_{0}\right)\right), \quad S\left(t_{0}\right)=0 .
$$

So, (2.1) is true. Moreover, by the positivity of the initial datum and $N(t)$, we get (2.2) from (1.2)-(1.4), which contradicts (2.1). Hence, it is impossible that $N(t)$ is positive but not $S(t)$. Step 3: We prove that it is impossible that $S(t)$ is positive but not $N(t)$.

Suppose that $S(t)$ is positive but not $N(t)$. Then $N(t)$ has one zero point in $[0,+\infty)$ at least. Let $t_{1}$ be the first zero point of $N(t)$ in $[0,+\infty)$. Then $N(0)>0$ implies that $t_{1}>0$ and

$$
N(t)>0 \quad\left(\forall t \in\left[0, t_{1}\right)\right), \quad N\left(t_{1}\right)=0 .
$$

Thus, we get (2.3). On the other hand, by the positivity of the initial datum and $S(t)$, we have (2.2) from (1.2)-(1.4), which contradicts (2.4). Therefore, it is impossible that $S(t)$ is positive but not $N(t)$.

In conclusion, if $(S(t), N(t))$ is a solution of (1.1)-(1.2) for a positive initial datum, then $S(t)$ and $N(t)$ are positive.

Theorem 2.2 Let $(S(t), N(t))$ be a solution of (1.1)-(1.2) for a positive initial datum. If $N(t)$ is bounded, then so is $S(t)$. 
Proof By Theorem 2.1, we know that $(S(t), N(t))$ is positive.

Multiplying two sides of (1.1) by $e^{\delta t}$, we have

$$
e^{\delta t} S^{\prime}(t)=-e^{\delta t} \delta S(t)+e^{\delta(t-\tau)} \beta(S(t-\tau)) f(N(t-\tau)), \quad t \geq 0,
$$

that is,

$$
\left(e^{\delta t} S(t)\right)^{\prime}=e^{\delta(t-\tau)} \beta(S(t-\tau)) f(N(t-\tau)), \quad t \geq 0 .
$$

Hence,

$$
e^{\delta t} S(t)-e^{\delta \tau} S(\tau)=\int_{\tau}^{t} e^{\delta(s-\tau)} \beta(S(s-\tau)) f(N(s-\tau)) d s, \quad t \geq \tau .
$$

Thus, for $t \geq \tau$,

$$
S(t)=e^{-\delta(t-\tau)} S(\tau)+e^{-\delta t} \int_{\tau}^{t} e^{\delta(s-\tau)} \beta(S(s-\tau)) f(N(s-\tau)) d s
$$

If $N(t)$ is bounded, that is, there is a constant $M>0$ such that

$$
0 \leq N(t) \leq M \quad(\forall t \geq 0)
$$

then it follows from (2.5), (1.4), and (2.6) that

$$
0 \leq S(t) \leq S(\tau)+\beta(0) L M \frac{e^{-\delta \tau}-e^{-\delta t}}{\delta} \leq S(\tau)+\beta(0) L M \frac{e^{-\delta \tau}}{\delta}, \quad t \geq \tau
$$

since $\beta$ is decreasing. This means that $S(t)$ is bounded.

Theorem 2.3 Let $(S(t), N(t))$ be a solution of (1.1)-(1.2) for a positive initial datum, and $\lim _{t \rightarrow+\infty} N(t)=0$. Then $\lim _{t \rightarrow+\infty} S(t)=0$.

Proof From $\lim _{t \rightarrow+\infty} N(t)=0$, it follows that (2.6) holds for some positive constant $M$.

By using (1.4), (2.6) and the condition that $\beta(\cdot)$ is a continuously differentiable, positive, and decreasing function, we deduce that for $t \geq \tau$,

$$
\begin{aligned}
S(t)= & e^{-\delta t}\left(e^{\delta \tau} S(\tau)+\int_{\tau}^{\frac{t+\tau}{2}} e^{\delta(s-\tau)} \beta(S(s-\tau)) f(N(s-\tau)) d s\right) \\
& +e^{-\delta t} \int_{\frac{t+\tau}{2}}^{t} e^{\delta(s-\tau)} \beta(S(s-\tau)) f(N(s-\tau)) d s \\
\leq & e^{-\delta t}\left(e^{\delta \tau} S(\tau)+L M \beta(0) e^{\delta\left(\frac{t-\tau}{2}\right)} \frac{t-\tau}{2}\right) \\
& +e^{-\delta t} L \beta(0) \int_{\frac{t+\tau}{2}}^{t} e^{\delta(s-\tau)} N(s-\tau) d s \\
\leq & e^{-\delta(t-\tau)} S(\tau)+L M \beta(0) e^{-\delta\left(\frac{t+\tau}{2}\right)} \frac{t-\tau}{2}
\end{aligned}
$$




$$
\begin{aligned}
& +e^{-\delta t} L \beta(0) N(\xi-\tau) \int_{\frac{t+\tau}{2}}^{t} e^{\delta(s-\tau)} d s \quad \text { by the mean value theorem } \\
\leq & e^{-\delta(t-\tau)} S(\tau)+L M \beta(0) e^{-\delta\left(\frac{t+\tau}{2}\right)} \frac{t-\tau}{2} \\
& +L \beta(0) \frac{e^{-\delta \tau}-e^{-\delta \frac{t+\tau}{2}}}{\delta} N(\xi-\tau),
\end{aligned}
$$

where $\xi \in\left[\frac{t+\tau}{2}, t\right]$.

Letting $t \rightarrow+\infty$ on both sides of the above inequality and noting that $\delta>0$ and

$$
\lim _{t \rightarrow+\infty} N(t)=0, \quad \lim _{t \rightarrow+\infty} e^{-\delta\left(\frac{t+\tau}{2}\right)} \frac{t-\tau}{2}=0,
$$

we have

$$
\lim _{t \rightarrow+\infty} S(t)=0 .
$$

Theorem 2.4 Let $(S(t), N(t))$ be a solution of (1.1)-(1.2) for a positive initial datum, and

$$
Y(t)=N(t)+2 e^{-\delta \tau} \int_{t-\tau}^{t} \beta(S(s)) f(N(s)) d s, \quad t \geq \tau .
$$

Assume that

$$
\left(2 e^{-\delta \tau}-1\right) \beta(0) L<\delta
$$

Then

$$
Y(t) \geq 0, \quad t \geq \tau
$$

and

$$
Y^{\prime}(t) \leq 0, \quad t \geq \tau
$$

Proof By Theorem 2.1, we see that

$$
S(t)>0, \quad N(t)>0, \quad t \geq 0 .
$$

Hence, (2.9) is true.

By (1.2) and (2.7), we have

$$
\begin{aligned}
Y^{\prime}(t) & =N^{\prime}(t)+2 e^{-\delta \tau}[\beta(S(t)) f(N(t))-\beta(S(t-\tau)) f(N(t-\tau))] \\
& =-\delta N(t)-\beta(S(t)) f(N(t))+2 e^{-\delta \tau} \beta(S(t)) f(N(t)) \\
& =-\delta N(t)+\left(2 e^{-\delta \tau}-1\right) \beta(S(t)) f(N(t)) .
\end{aligned}
$$

Case 1: $2 e^{-\delta \tau}-1 \leq 0$. 
In this case, by the property of $\beta,(2.11),(1.4)$ and (2.12), we get

$$
Y^{\prime}(t)=-\delta N(t)+\left(2 e^{-\delta \tau}-1\right) \beta(S(t)) f(N(t)) \leq 0, \quad t \geq \tau .
$$

Case 2: $2 e^{-\delta \tau}-1>0$.

In this case, by the property of $\beta,(2.11),(1.4),(2.12)$ and (2.8), we obtain

$$
\begin{aligned}
Y^{\prime}(t) & =-\delta N(t)+\left(2 e^{-\delta \tau}-1\right) \beta(S(t)) f(N(t)) \\
& \leq-\delta N(t)+\left(2 e^{-\delta \tau}-1\right) \beta(0) L N(t) \\
& =\left[\left(2 e^{-\delta \tau}-1\right) \beta(0) L-\delta\right] N(t) \\
& <0, \quad t \geq \tau .
\end{aligned}
$$

Combining the two cases above, we know that if (2.8) holds, then

$$
Y^{\prime}(t) \leq 0, \quad t \geq \tau
$$

Theorem 2.5 Let $(S(t), N(t))$ be a solution of (1.1)-(1.2) for a positive initial datum, and $Y(t)$ be given by (2.7). Assume that (2.8) holds. Then

$$
\lim _{t \rightarrow+\infty} Y^{\prime}(t)=0
$$

Proof It follows from Theorem 2.4 that $Y(t)$ is decreasing and lower bounded by 0 for $t \geq \tau$. So, $\inf _{t \geq \tau} Y(t)$ exists.

Set

$$
y_{0}=\inf _{t \geq \tau} Y(t)
$$

Then, it is clear that $y_{0} \geq 0$ by (2.9), and for any $\varepsilon>0$, there exists $T_{0}>\tau$ such that $Y\left(T_{0}\right)<$ $y_{0}+\varepsilon$. Since $Y(t)$ is a decreasing function, for every $t>T_{0}$,

$$
y_{0}-\varepsilon<y_{0} \leq Y(t) \leq Y\left(T_{0}\right)<y_{0}+\varepsilon \text {. }
$$

This implies that

$$
\lim _{t \rightarrow+\infty} Y(t)=y_{0}
$$

Hence, $Y(t)$ is bounded on $[\tau,+\infty)$. Consequently, $N(t)$ is bounded on $[0,+\infty)$, and Theorem 2.2 shows that $S(t)$ is also bounded on $[0,+\infty)$.

Moreover, it follows from (1.1) and (1.2) that $N^{\prime}(t)$ and $S^{\prime}(t)$ are bounded on $[0,+\infty)$. Thus, $N(t)$ and $S(t)$ are Lipschitz continuous on $[0,+\infty)$, and consequently $N(t)$ and $S(t)$ are uniformly continuous on $[0,+\infty)$. Combining these facts with $(2.14)$ and the continuity of $\beta$ and $f$, we know that

$Y^{\prime}(t)$ is bounded and uniformly continuous on $[\tau,+\infty)$. 
By virtue of (2.16), we know that (2.15) is true, owing to the following known result by Barbălat (see Gopalsamy [15]):

Let $g:[a,+\infty) \rightarrow R, a \in R$, be a differentiable function. If $\lim _{t \rightarrow+\infty} g(t)$ exists and $g^{\prime}(t)$ is uniformly continuous on $(a,+\infty)$, then

$$
\lim _{t \rightarrow+\infty} g^{\prime}(t)=0 .
$$

Theorem 2.6 Let $(S(t), N(t))$ be a solution of (1.1)-(1.2) for a positive initial datum, and let $(2.8)$ hold. Then $(S(t), N(t))$ tends to $(0,0)$.

Proof First, we prove that

$$
\lim _{t \rightarrow+\infty} N(t)=0
$$

Actually, if this is not true, then there exists an $\varepsilon_{0}>0$ such that for every positive integer $n$, there is $t_{n}>n$ such that

$$
N\left(t_{n}\right)>\varepsilon_{0} .
$$

By the boundedness of $N(t)$ on $[0,+\infty)$, we infer that there exists a subsequence $\left\{t_{n_{k}}\right\}$ of $\left\{t_{n}\right\}$ such that

$$
t_{n_{k}}>\tau \text { for all } k>k_{0} \text {, }
$$

where $k_{0}$ is a fixed positive integer, and

$$
\lim _{k \rightarrow+\infty} N\left(t_{n_{k}}\right)=\alpha \geq \varepsilon_{0}>0
$$

where $\alpha$ is a constant. Therefore, we have the following observations.

Case 1: $2 e^{-\delta \tau}-1 \leq 0$.

In this case, by (2.13) we get

$$
\lim _{k \rightarrow+\infty} Y^{\prime}\left(t_{n_{k}}\right) \leq-\delta \lim _{k \rightarrow+\infty} N\left(t_{n_{k}}\right)=-\delta \alpha<0,
$$

which contradicts (2.15).

Case 2: $2 e^{-\delta \tau}-1>0$.

In this case, by the property of $\beta,(2.11),(1.4),(2.12)$ and (2.8), we have

$$
\begin{aligned}
\lim _{k \rightarrow+\infty} Y^{\prime}\left(t_{n_{k}}\right) & \leq \lim _{k \rightarrow+\infty}\left[-\delta N\left(t_{n_{k}}\right)+\left(2 e^{-\delta \tau}-1\right) \beta(0) L N\left(t_{n_{k}}\right)\right] \\
& =\lim _{k \rightarrow+\infty}\left[\left(2 e^{-\delta \tau}-1\right) \beta(0) L-\delta\right] N\left(t_{n_{k}}\right) \\
& =\alpha\left[\left(2 e^{-\delta \tau}-1\right) \beta(0) L-\delta\right] \\
& <0,
\end{aligned}
$$

which also contradicts (2.15).

Consequently, (2.17) holds. This, together with Theorem 2.1, shows that

$$
\lim _{t \rightarrow+\infty} S(t)=0
$$




\section{Numerical simulations}

With Matlab software we give two numerical simulations of the main result, Theorem 2.6.

Figure 1, where $S(t)$ is the right (blue) curve and $N(t)$ is the left (green) curve, is plotted with the following case for $\tau=0.5$ of (1.1)-(1.2)

$$
\begin{aligned}
S^{\prime}(t)= & -S(t)+\frac{e^{-0.5}}{1+10^{-12} S(t-0.5)^{1.25}} \times \frac{\lambda N(t-0.5)}{1+4 \times 10^{-8} N(t-0.5)}, \quad t \geq 0, \\
N^{\prime}(t)= & -N(t)-\frac{\lambda N(t)}{\left(1+10^{-12} S(t)^{1.25}\right)\left(1+4 \times 10^{-8} N(t)\right)} \\
& +\frac{2 e^{-0.5}}{1+10^{-12} S(t-0.5)^{1.25}} \times \frac{\lambda N(t-0.5)}{1+4 \times 10^{-8} N(t-0.5)}, \quad t \geq 0,
\end{aligned}
$$

with $\lambda=0.60653 e^{0.5}$.

For the following case for $\tau=0.4$ of (1.1)-(1.2),

$$
\begin{aligned}
S^{\prime}(t)= & -S(t)+\frac{e^{-0.4}}{1+10^{-12} S(t-0.4)^{1.25}} \times \frac{\lambda N(t-0.4)}{1+4 \times 10^{-8} N(t-0.4)}, \quad t \geq 0, \\
N^{\prime}(t)= & -N(t)-\frac{\lambda N(t)}{\left(1+10^{-12} S(t)^{1.25}\right)\left(1+4 \times 10^{-8} N(t)\right)} \\
& +\frac{2 e^{-0.4}}{1+10^{-12} S(t-0.4)^{1.25}} \times \frac{\lambda N(t-0.4)}{1+4 \times 10^{-8} N(t-0.4)}, \quad t \geq 0,
\end{aligned}
$$

with $\lambda=0.60653 e^{0.4}$, we have Figure 2 , where $S(t)$ is the right (blue) curve and $N(t)$ is the left (green) curve.

Both numerical simulations illustrate our result very well and show that for these cases, the hematopoietic stem cell population is definitely extinct.

In this paper, we investigated the asymptotic behavior of solutions of some nonlinear delay models of hematopoietic stem cell dynamics. The nonlinearity depends upon the entire hematopoietic stem cell population as well as the nonlinear change of the number

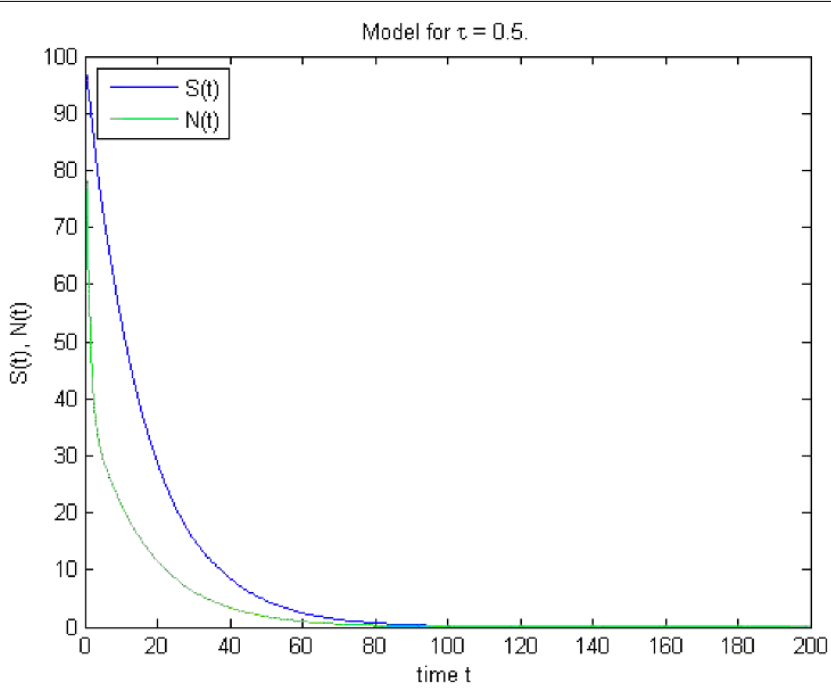

Figure 1 Simulations of solutions of the model for $\boldsymbol{\tau}=0.5$. 


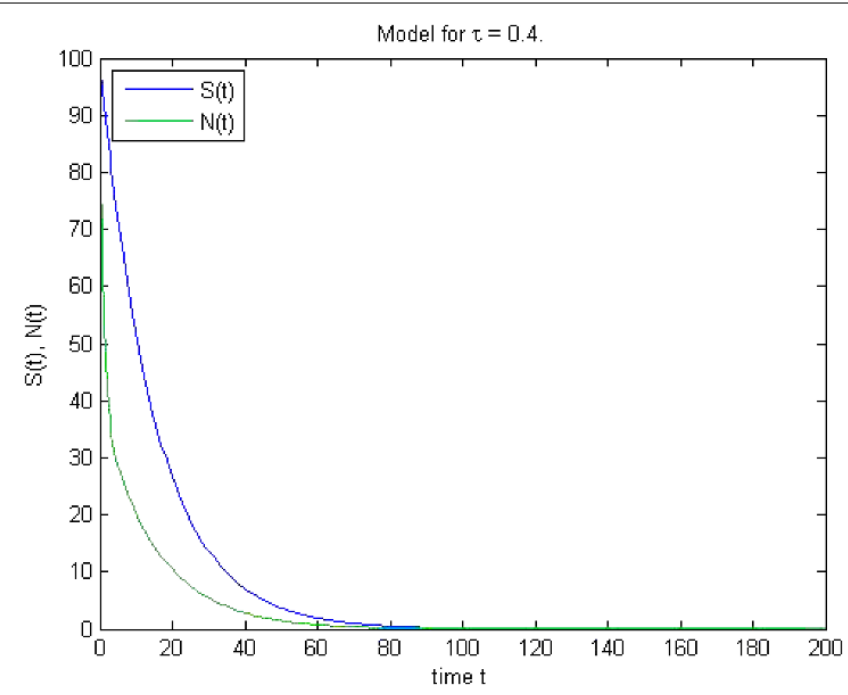

Figure 2 Simulations of solutions of the model for $\tau=0.4$.

of nonproliferating cell compartments, which is different from the models considered in the previous literature on blood cell production models ( $c f$. [7] and references therein). Moreover, this class of models covers essentially those in the previous works. By using arguments different from [7], we proved Theorems 2.1 and 2.3. By employing a new analysis process, we overcame the difficulty caused by the nonlinear term $f$ and proved Theorem 2.6. As special cases, we can deduce the corresponding results given in these works from Theorems 2.1-2.6. Finally, by giving a numerical investigation, we illustrated efficiently the asymptotic stability of the solutions to some models of the cell population dynamics. For further analysis, the stability of the models with appropriate feedback controls is a good issue.

\section{Competing interests}

The author declares that they have no competing interests.

\section{Acknowledgements}

I would like to thank the anonymous referees and Prof. Z Lei for their valuable comments and suggestions.

Received: 17 February 2013 Accepted: 27 June 2013 Published: 11 July 2013

\section{References}

1. Simmons, A: Basic Hematology. Charles C Thomas, Springfield (1973)

2. Israels, L, Israels, E: Erythropoiesis: an overview. In: Erythropoietins and Erythropoiesis: Molecular, Cellular, Preclinical and Clinical Biology, pp. 3-14. Birkhäuser, Basel (2003)

3. Bliss, KM: Modeling of red blood cell dynamics in patients with chronic kidney disease. PhD thesis, North Carolina State University (2011)

4. Mackey, MC: Unified hypothesis of the origin of aplastic anaemia and periodic hematopoiesis. Blood 51, 941-956 (1978)

5. Mackey, MC: Cell kinetic status of haematopoietic stem cells. Cell Prolif. 34, 71-83 (2001)

6. Mackey, MC, Glass, L: Oscillation and chaos in physiological control systems. Science 197, $287-289$ (1977)

7. Crauste, F: Delay model of hematopoietic stem cell dynamics: asymptotic stability and stability switch. Math. Model. Nat. Phenom. 4, 28-47 (2009)

8. Drobnjak, I, Fowler, AC: A model of oscillatory blood cell counts in chronic myelogenous leukaemia. Bull. Math. Biol. 73, 2983-3007 (2011)

9. Iannelli, M: Mathematical Theory of Age-Structured Population Dynamics. Applied Mathematics Monograph C.N.R. vol. 7. Giardini Editori E Stampatori, Pisa (1995)

10. Perthame, B: Transport Equations in Biology. Birkhäuser, Basel (2007)

11. Cuevas, C, Frasson, MVS: Asymptotic properties of solutions to linear nonautonomous delay differential equations through generalized characteristic equations. Electron. J. Differ. Equ. 2010, 95 (2010) 
12. Cuevas, C, Vidal, C: A note on discrete maximal regularity for functional difference equations with infinite delay. Adv. Differ. Equ. 2006, Article ID 97614 (2006)

13. Diagana, T: Weighted pseudo-almost periodic solutions to a neutral delay integral equation of advanced type. Nonlinear Anal. 70, 298-304 (2009)

14. Diagana, T, Hernández, E, Rabello, M: Pseudo almost periodic solutions to some non-autonomous neutral functional differential equations with unbounded delay. Math. Comput. Model. 45, 1241-1252 (2007)

15. Gopalsamy, K: Stability and Oscillations in Delay Differential Equations of Population Dynamics. Mathematics and Its Applications, vol. 74. Kluwer Academic, Dordrecht (1992)

doi:10.1186/1687-1847-2013-207

Cite this article as: Liang: Asymptotic behavior of solutions of nonlinear models from erythropoiesis. Advances in Difference Equations 2013 2013:207.

Submit your manuscript to a SpringerOpen ${ }^{\circ}$ journal and benefit from:

- Convenient online submission

- Rigorous peer review

Immediate publication on acceptance

- Open access: articles freely available online

- High visibility within the field

- Retaining the copyright to your article

Submit your next manuscript at $>$ springeropen.com 